\title{
THE 2011 OUTBURST OF RECURRENT NOVA T Pyx: X-RAY OBSERVATIONS EXPOSE THE WHITE DWARF MASS AND EJECTION DYNAMICS
}

\author{
Laura Chomiuk ${ }^{1,2}$, Thomas Nelson ${ }^{3}$, Koji Mukai $^{4,5}$, J. L. Sokoloski ${ }^{6}$, Michael P. Rupen $^{2}$, \\ Kim L. Page ${ }^{7}$, Julian P. Osborne ${ }^{7}$, Erik Kunlkers ${ }^{8}$, Amy J. Mioduszewski ${ }^{2}$, \\ Nirupam Roy ${ }^{9}$, Jennifer Weston ${ }^{6}$, and Miriam I. Krauss ${ }^{2}$ \\ ${ }^{1}$ Department of Physics and Astronomy, Michigan State University, East Lansing, MI 48824, USA; chomiuk@ pa.msu.edu \\ ${ }^{2}$ National Radio Astronomy Observatory, P.O. Box O, Socorro, NM 87801, USA \\ ${ }^{3}$ School of Physics and Astronomy, University of Minnesota, 115 Church Street SE, Minneapolis, MN 55455, USA \\ ${ }^{4}$ CRESST and X-ray Astrophysics Laboratory, NASA/GSFC, Greenbelt, MD 20771, USA \\ ${ }^{5}$ Center for Space Science and Technology, University of Maryland Baltimore County, 1000 Hilltop Circle, Baltimore, MD 21250, USA \\ ${ }^{6}$ Columbia Astrophysics Laboratory, Columbia University, New York, NY, USA \\ ${ }^{7}$ Department of Physics and Astronomy, University of Leicester, Leicester, LE1 7RH, UK \\ ${ }^{8}$ European Space Astronomy Centre (ESA/ESAC), Science Operations Department, E-28691 Villanueva de la Caada, Madrid, Spain \\ ${ }^{9}$ Max Planck Institut für Radioastronomie, Auf dem Hügel 69, D-53121 Bonn, Germany \\ Received 2014 February 16; accepted 2014 May 2; published 2014 May 30
}

\begin{abstract}
The recurrent nova T Pyx underwent its sixth historical outburst in 2011, and became the subject of an intensive multi-wavelength observational campaign. We analyze data from the Swift and Suzaku satellites to produce a detailed $\mathrm{X}$-ray light curve augmented by epochs of spectral information. X-ray observations yield mostly non-detections in the first four months of outburst, but both a super-soft and hard X-ray component rise rapidly after Day 115. The super-soft X-ray component, attributable to the photosphere of the nuclear-burning white dwarf, is relatively $\operatorname{cool}(\sim 45 \mathrm{eV})$ and implies that the white dwarf in T Pyx is significantly below the Chandrasekhar mass $\left(\sim 1 M_{\odot}\right)$. The late turn-on time of the super-soft component yields a large nova ejecta mass $\left(\gtrsim 10^{-5} M_{\odot}\right)$, consistent with estimates at other wavelengths. The hard X-ray component is well fit by a $\sim 1 \mathrm{keV}$ thermal plasma, and is attributed to shocks internal to the 2011 nova ejecta. The presence of a strong oxygen line in this thermal plasma on Day 194 requires a significantly super-solar abundance of oxygen and implies that the ejecta are polluted by white dwarf material. The X-ray light curve can be explained by a dual-phase ejection, with a significant delay between the first and second ejection phases, and the second ejection finally released two months after outburst. A delayed ejection is consistent with optical and radio observations of T Pyx, but the physical mechanism producing such a delay remains a mystery.
\end{abstract}

Key words: novae, cataclysmic variables - stars: individual (T Pyxidis) - white dwarfs - X-rays: stars

Online-only material: color figures

\section{INTRODUCTION}

The five thermonuclear explosions of T Pyxidis observed in $1890,1902,1920,1944$, and 1966 earned the system its place as the prototypical recurrent nova, but have also highlighted our poor understanding of many aspects of binary evolution and nova theory. The community has waited anxiously for the next outburst of T Pyx in order to study this peculiar system with modern multi-wavelength capabilities, and T Pyx finally obliged by entering its sixth recorded outburst in 2011 April. High-quality panchromatic observations are now revealing a host of new surprises for this system.

A nova is a transient event marking a thermonuclear runaway on the surface of an accreting white dwarf. The white dwarf accretes hydrogen-rich material from a companion star, and this accreted material settles down into a thin degenerate layer on the surface of the white dwarf. The pressure and temperature in this layer increase until explosive nuclear burning begins, and the bulk of the accreted envelope is expelled from the white dwarf at hundreds to thousands of $\mathrm{km} \mathrm{s}^{-1}$.

Novae are expected to recur on an accreting white dwarf with a timescale primarily determined by the white dwarf mass and accretion rate (e.g., Yaron et al. 2005; Wolf et al. 2013). Predicted recurrence timescales vary widely $\left(\sim 1-10^{8} \mathrm{yr}\right.$; Yaron et al. 2005), and those novae repeating on historical timescales have been dubbed "recurrent" novae. Theoretically, we expect recurrent novae to occur in binaries where massive white dwarfs accrete at high rates, because more massive white dwarfs have higher surface gravities, meaning that the critical conditions are reached for smaller accreted envelopes, and higher accretion rate systems accrue this trigger mass in less time.

Even before 2011, T Pyx flew in the face of our expectations for recurrent novae. The evolution of its optical light curve is slow, showing a several months-long plateau around maximum light and a relatively slow decline from this maximum (Schaefer 2010). T Pyx has a short orbital period $(1.83 \mathrm{hr}$; Uthas et al. 2010), solidly below the cataclysmic-variable (CV) period gap. According to the theory of $\mathrm{CV}$ evolution, such short-period systems should have, on average, very low accretion rates (e.g., Knigge 2011), but observations in quiescence-and the short nova recurrence time-imply that $\mathrm{T}$ Pyx has an accretion rate orders of magnitude higher than these expectations (Gilmozzi \& Selvelli 2007; Selvelli et al. 2008). In addition, measured binary parameters imply that the white dwarf in T Pyx may be significantly less massive than the Chandrasekhar mass (Uthas et al. 2010), in contrast with common assumptions for recurrent novae.

The high accretion rate in $\mathrm{T}$ Pyx does not appear to be sustainable, as it is exceeds expectations by several orders 
of magnitude for mass transfer rates driven by gravitational radiation (the commonly accepted mass transfer mechanism at such short orbital periods). Knigge et al. (2000) and Schaefer et al. (2010) have hypothesized that mass transfer in T Pyx is in a short-term elevated state, perhaps incited by a powerful nova outburst which occurred during the 1800s (before regular records were kept on T Pyx). Before this postulated event, T Pyx may have been a typical CV below the period gap, with a very low accretion rate and long intervals between novae. However, after the hypothesized explosion, the hot white dwarf irradiated the companion star and induced an unusually high mass transfer rate. Perhaps this irradiation power is slowly dwindling and the accretion rate is gradually declining, explaining the increasing intervals between nova events observed for T Pyx throughout the last century. Observational tests of this hypothesis have reached divergent conclusions as to whether there is evidence for a secular decline in T Pyx's accretion rate (e.g., Schaefer et al. 2013; Godon et al. 2014).

Regardless of T Pyx's history, it is clear that T Pyx provides an opportunity to test an unusual corner of nova parameter space. Compared with other novae, we have a thorough understanding of the binary system's parameters and the accretion rate (Selvelli et al. 2008; Uthas et al. 2010). With multiwavelength data collected from the 2011 outburst, we can measure key properties of the nova event, like ejected mass, and compare them with predictions from nova models. With this goal in mind, campaigns have been carried out across the entire electromagnetic spectrum, providing an exquisitely detailed picture of the 2011 nova outburst of T Pyx (Chesneau et al. 2011; Kuulkers et al. 2011a, 2011b; Shore et al. 2011, 2013; Evans et al. 2012; Imamura \& Tanabe 2012; Nelson et al. 2014; Ederoclite 2013; Williams 2013; Schaefer et al. 2013; Patterson et al. 2013; Sokoloski et al. 2013; Tofflemire et al. 2013; De Gennaro Aquino et al. 2014; Godon et al. 2014; Surina et al. 2014).

At every wavelength studied so far, the 2011 outburst of T Pyx shows surprising features when compared to expectations for recurrent novae. As in previous outbursts, the optical light curve shows a sort of plateau for three months (Figure 1), implying that the optical photosphere is roughly constant in size for $\sim 90$ days after thermonuclear runaway (Shore et al. 2013). Optical spectroscopy shows that T Pyx is an unusual "hyperhybrid" nova, switching from $\mathrm{He} / \mathrm{N}$ class to Fe II class around Day 10, and then back to He/N on Day 65 (Williams 2012, 2013; Ederoclite 2013; Surina et al. 2014). The radio light curve and optical measurements of the change in the binary period after the nova event imply a large ejected mass $\left(\sim 10^{-4}-10^{-5} M_{\odot}\right.$; Nelson et al. 2014; henceforth, Paper I; Patterson et al. 2013), rather than the $\sim 10^{-6}-10^{-7} M_{\odot}$ expected for recurrent novae. Radio light curves also show a late and steep rise (Figure 1; Paper I), implying that either the ejecta in T Pyx were very cold $(<200 \mathrm{~K})$ during the first $\sim 50$ days of the outburst, or the bulk of the nova ejecta stalled at an $\sim$ AU-scale radius until it was finally expelled $\sim 50$ days after the thermonuclear runaway (such a delay might also explain the long plateau in the optical light curve; Shore et al. 2013).

In this work, we focus on X-ray observations of the 2011 outburst of T Pyx obtained with Swift and Suzaku, and compare our results with inferences from other wavelengths. X-ray emission from novae can be split into two broad classes, which may, but need not, exist contemporaneously (Krautter 2008). The first class is super-soft X-ray emission, characterized by effective temperatures between $10^{5}$ and $10^{6} \mathrm{~K}$, and luminosities

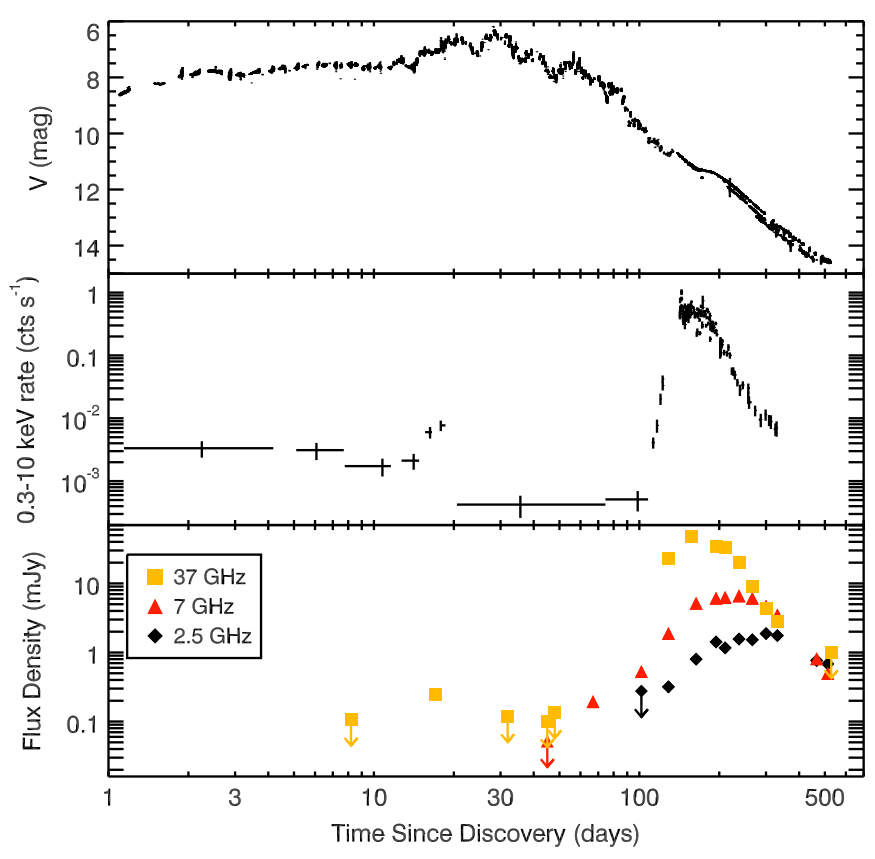

Figure 1. Overview of the 2011 outburst of T Pyx at optical, X-ray, and radio wavelengths. Top: $V$-band optical data from the AAVSO. Middle: $0.3-10 \mathrm{keV}$ $\mathrm{X}$-ray light curve obtained with $S$ wift/XRT. Bottom: VLA radio light curve at $2.5,7$, and $37 \mathrm{GHz}$ (Paper I).

(A color version of this figure is available in the online journal.)

in the range $10^{36}-10^{38} \mathrm{erg} \mathrm{s}^{-1}$. High-resolution spectra obtained with the grating instruments onboard Chandra and XMMNewton have confirmed that this emission originates near the white dwarf photosphere (Nelson et al. 2008; Rauch et al. 2010; Ness et al. 2011; Orio 2012). In some novae (e.g., V2491 Cyg, $\mathrm{RS}$ Oph) the soft X-ray flux is continuum emission that most likely originates at the white dwarf photosphere. In other cases, the soft X-rays are associated with strong lines of $\mathrm{H}$ and $\mathrm{He}-$ like carbon, nitrogen and oxygen that likely indicate scattered photospheric emission (see Ness et al. 2013 and references therein). Super-soft X-ray emission only becomes visible at later stages of the nova outburst, once the ejecta have become optically thin to the radiation from the hot, still burning white dwarf surface layers; therefore, the emergence time of the supersoft source can be used as a diagnostic for ejecta mass (Henze et al. 2011; Schwarz et al. 2011).

Observations of novae in the $\sim 1-10 \mathrm{keV}$ energy range also reveal harder X-ray emission on timescales of days to years after outburst (e.g., Mukai et al. 2008). During the novae in RS Oph and V407 Cyg, both of which have red giant secondaries, hard X-ray emission was detected at early times and attributed to the interaction of the nova ejecta with the dense wind of the companion (Sokoloski et al. 2006; Nelson et al. 2012). In systems with less evolved donors (and hence lower density circumbinary environments), internal shocks within the ejecta have been proposed as the origin for hard X-ray emission (see Mukai et al. 2008 and references therein). In these cases, hard $\mathrm{X}$-ray emission can tell us about the structure of the ejecta as the nova outburst progresses (O’Brien et al. 1994). In the last three years, novae have been identified as a new class of GeV gamma-ray transient by Fermi/LAT, indicating that the shock interactions in novae are capable of accelerating particles to relativistic speeds (Abdo et al. 2010; Hill et al. 2013; see also Tatischeff \& Hernanz 2007). Observations of novae in the $1-10 \mathrm{keV}$ range are required to characterize these shocks 
and fully understand the gamma-ray production mechanism. We note that T Pyx was not detected with Fermi (C. Cheung 2013, private communication), making it a useful comparison case for the study of why some novae produce detectable gamma-rays while others do not.

In this paper, we discuss both modes of X-ray emission during the 2011 outburst of T Pyx. In Section 2, we discuss the $\mathrm{X}$-ray observations and data reduction; Swift monitoring reveals the X-ray evolution at high cadence, while our single epoch of Suzaku spectroscopy provides high signal to noise on Day 194. In Section 3, we describe the Swift X-ray light curve, and Section 4 presents our spectral analysis of the Swift and Suzaku data. Section 5 analyzes the observed hard X-ray component and concludes that it is likely produced by a shock within the ejecta, rather than interaction between the nova and preexisting circumbinary material. Section 6 presents a super-soft component with a relatively cool temperature and late turnon time (compared to other recurrent novae). In Section 7, we discuss how these X-ray results align with optical and radio observations, and suggest that all three wavelength regimes support a second, massive, delayed ejection in $\mathrm{T}$ Pyx. We conclude in Section 8.

Throughout the paper, we take 2011 April $14(\mathrm{MJD}=55665)$ as $t_{0}$ or Day 0 , the beginning of optical rise and the start of the outburst (Waagan et al. 2011; Schaefer et al. 2013). We also assume a distance to T Pyx of $4.8 \pm 0.5 \mathrm{kpc}$ (Sokoloski et al. 2013).

\section{OBSERVATIONS AND DATA REDUCTION}

\subsection{Swift}

The 2011 outburst of T Pyx was monitored frequently with the Swift satellite as part of the ongoing monitoring of novae by the Swift Nova CV Group (Kuulkers et al. 2011a, 2011b; Osborne et al. 2011a). A series of 221 Swift X-ray Telescope (XRT) observations of T Pyx were carried out between 2011 April 14 (Day 1) and 2012 April 17 (Day 369), resulting in one of the most detailed X-ray light curves of a nova obtained to date. All observations were made in photon counting mode. The total XRT exposure time was $\sim 271 \mathrm{ks}$, with a median snapshot duration of $\sim 1 \mathrm{ks}$. Swift observed T Pyx daily during the first month of the outburst, and then reduced the cadence to 2-3 times per week for the subsequent three months. Once the X-ray emission became bright at the start of 2011 September (see Section 3 below), the cadence was once more increased to daily, and remained so for the next two months. The observing cadence was reduced at late times, ultimately resulting in weekly observations after 2012 January, through 2012 April (Days 262-369).

We created grade 0-12 event light curves and time-resolved hardness ratios using the Swift/XRT products generator developed by the Swift group at the University of Leicester. ${ }^{10}$ This web-based tool can create X-ray light curves, spectra and images of any object that has been observed with the XRT, and makes use of tools originally developed for automatic reduction of gamma-ray burst observations. Details of these products are given in Evans et al. (2009). We used an adaptive binning strategy to maximize the count rate and hardness ratio information in the light curves. Prior to Day 124, and after Day 200, the data were binned to give a minimum of 20 counts per bin. In between these days while the source was bright, the data were binned by observation (i.e., all snapshots with a single

\footnotetext{
10 http://www.swift.ac.uk/user_objects/
}

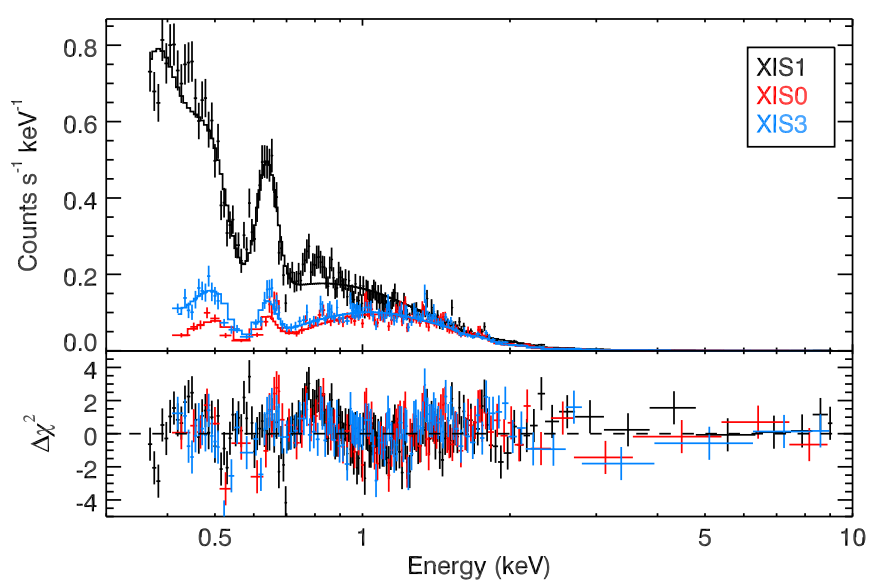

Figure 2. X-ray spectrum of T Pyx as observed with Suzaku on Day 194 in three different XIS units (colored as black, red, and blue lines). The data are shown in the upper panel as points, overlaid with the best-fit model (the sum of a blackbody, a thermal bremsstrahlung plasma, and a Gaussian line) in solid lines. The parameters of this model can be found in Table 1. Residuals in the fit to the data are shown in units of sigma in the lower panel. The data shown here are without the energy offset discussed in Section 4.1, to illustrate the offset in energy calibration between XIS1 and XIS0/XIS3.

(A color version of this figure is available in the online journal.)

observation ID). We also created spectra for certain time intervals during the outburst (see Section 4.2), again using the XRT products generator. We obtained the appropriate response matrix file, in this case swxpc0to12s6_20010101v013.rmf, from the calibration database. The resulting spectra were binned to have a minimum of one count per bin, and then modeled in XSpec using the Cash statistic, a maximum likelihood-based statistic for Poisson data (Cash 1979).

\subsection{Suzaku}

Given the X-ray rise reported by Osborne et al. (2011a), we requested and were granted a Director's Discretionary Time observation of T Pyx with the Suzaku observatory. The exposure was carried out on 2011 October 25 (Day 194). Here, we focus on the data obtained with the X-ray Imaging Spectrometer (XIS) in the $0.3-10 \mathrm{keV}$ energy range. All three functioning XIS units were operated in the full-window imaging mode, obtaining X-ray event data every 8 s over the full $19^{\prime}$ by $19^{\prime}$ field of view. After standard screening, ${ }^{11}$ the net exposure time was $\sim 38,600$ s for each XIS unit. We extracted the source spectra from a $180^{\prime \prime}$ region centered on T Pyx using XSelect v2.4b. Background spectra were extracted from annular regions also centered on the source, with inner radius $240^{\prime \prime}$ and outer radius $420^{\prime \prime}$ for both XIS1 and XIS3, while the outer radius was 390" for XISO (the smaller size is to avoid dead regions of the chip). We created response files using the xisrmf gen and xisarfgen ftools. The most recent version of the contamination model (version 20120719) was used to calculate the effective areas. The resulting spectra were binned to have a minimum of 30 counts per bin in order to facilitate the use of $\chi^{2}$ statistics in determining the best-fit spectral model (Figure 2).

\section{X-RAY LIGHT CURVE OF THE 2011 OUTBURST OF T Pyx}

We present the Swift XRT light curve in the middle panel of Figure 1, as well as the $V$-band AAVSO optical light curve

\footnotetext{
11 http://heasarc.gsfc.nasa.gov/docs/suzaku/processing/criteria_xis.html?
} 

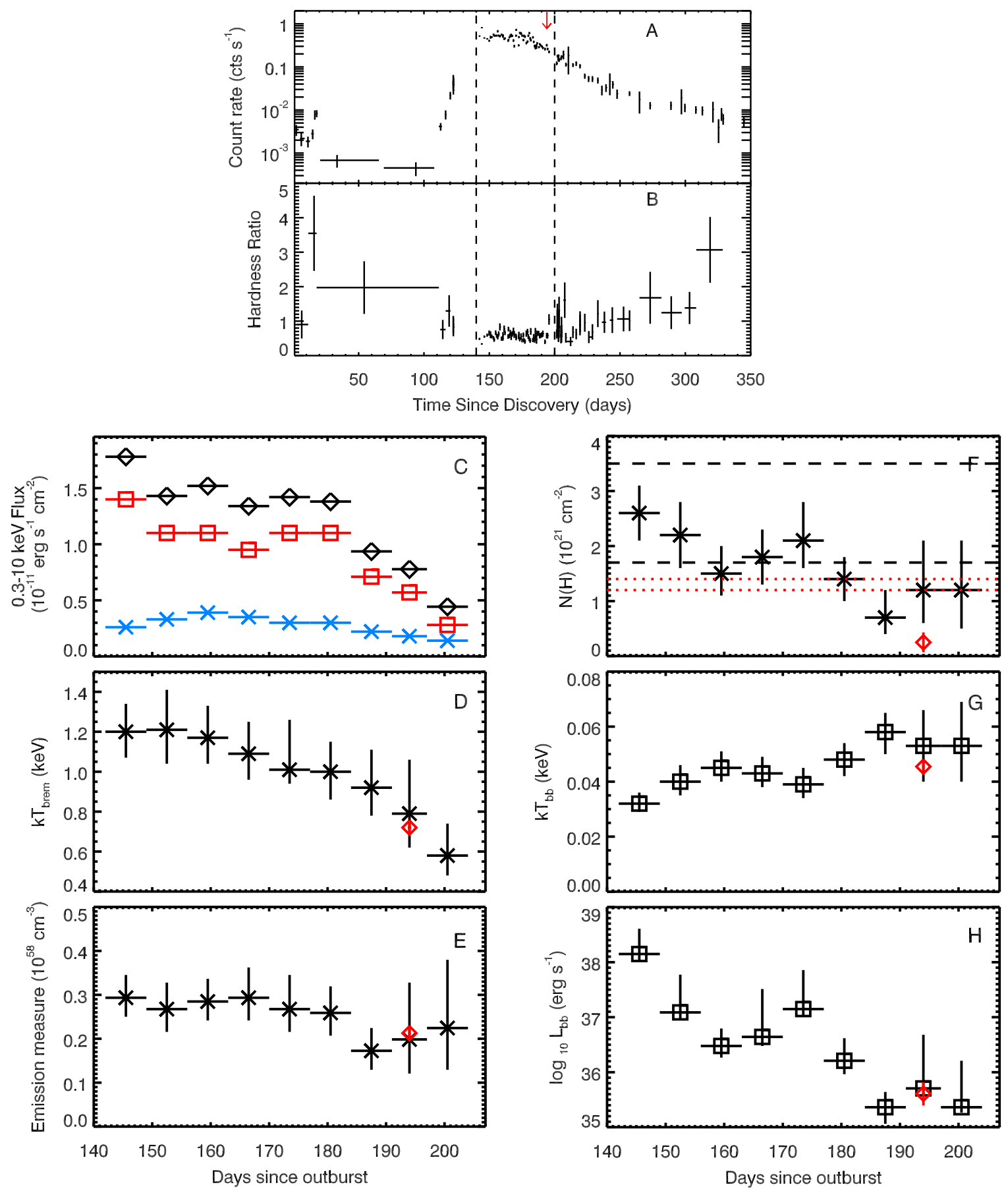

Figure 3. (a) Swift/XRT light curve of T Pyx in the $0.3-10 \mathrm{keV}$ energy range (as in Figure 1, this time with a linear time axis). The red arrow marks the time of the Suzaku observation, and the dashed vertical lines mark the period for which we analyze the Swift spectra. (b) X-ray hardness ratio (defined as the ratio of the $0.55-10 \mathrm{keV}$ to $0.3-0.55 \mathrm{keV}$ count rate) as a function of time. (c) X-ray flux in $0.3-10 \mathrm{keV}$ range derived from weekly averaged Swift spectra. Black diamonds show the total flux, red squares mark the blackbody component, and blue crosses denote the bremsstrahlung flux. (c) and (e) Temperature and volume emission measure evolution of the thermal plasma component. (f) The evolution of the absorbing column density. The range of interstellar absorbing column derived by Shore et al. (2011) is shown as black dashed lines, and the Gilmozzi \& Selvelli (2007) measurement is bracketed by red dotted lines. (g) and (h) The evolution of the blackbody temperature and luminosity. The best-fit values from the Suzaku spectrum are shown as red diamonds in panels (d)-(h).

(A color version of this figure is available in the online journal.)

(upper panel) and a three-frequency radio light curve obtained with the Karl G. Jansky Very Large Array (VLA) in the lower panel. The Swift XRT light is also plotted in Figure 3(a) with a linear time axis.

Individual X-ray observations during the first 100 days of the outburst mainly resulted in non-detections, with the notable exception of the detections between Days 14-20. T Pyx is detected as a faint hard X-ray source in this third week after outburst (Figure 3(b)), but then the X-rays fade out of detectability again for the next three months.

On Day 117, T Pyx was seen at 0.003 counts s ${ }^{-1}$, and by Day 125 it had increased in count rate by a factor of 10 . T Pyx then entered a sun-angle constraint, and was not observed again until 2011 September 3 (Day 142). This first visit back to T Pyx 
Table 1

Best-fit Model Parameters for Day 194 Suzaku Spectrum ${ }^{a}$

\begin{tabular}{|c|c|c|c|c|}
\hline & tbnew* (bb+brems+gauss) & tbnew* $(\mathrm{bb}+\text { vapec })_{a}$ & tbnew* $(\mathrm{bb}+\text { vapec })_{b}$ & tbnew* $(b b+v n e i)$ \\
\hline$\overline{N(H)\left(10^{21} \mathrm{~cm}^{-2}\right)}$ & $0.27 \pm 0.19$ & $2.0 \pm 0.02$ & $0.61 \pm 0.02$ & $0.5 \pm 0.02$ \\
\hline$k T_{\mathrm{BB}}(\mathrm{eV})$ & $47 \pm 2$ & $35 \pm 2$ & $44 \pm 2$ & $46 \pm 2$ \\
\hline $\operatorname{Norm}_{B B}{ }^{b}$ & $0.0016_{-0.0006}^{+0.0010}$ & $0.083_{-0.001}^{+0.002}$ & $0.0032 \pm 0.0020$ & $0.0026_{-0.0007}^{+0.0018}$ \\
\hline$k T_{\text {br }}(\mathrm{keV})$ & $0.72 \pm 0.03$ & $\ldots$ & $\ldots$ & $\ldots$ \\
\hline $\operatorname{Norm}_{b r}{ }^{c}\left(10^{-3}\right)$ & $2.5 \pm 0.2$ & $\ldots$ & $\ldots$ & $\ldots$ \\
\hline$k T_{\text {vapec }}(\mathrm{keV})$ & $\ldots$ & $0.70 \pm 0.02$ & $0.73 \pm 0.04$ & $\ldots$ \\
\hline Norm $_{\text {vapec }}{ }^{d}\left(10^{-3}\right)$ & $\ldots$ & $0.25 \pm 0.03$ & $7.4 \pm 0.5$ & $\ldots$ \\
\hline$Z / Z_{\odot}$ & $\ldots$ & 1.0 & $<0.003$ & $\ldots$ \\
\hline$O / O_{\odot}$ & $\cdots$ & $150_{-4}^{+6}$ & $1.6_{-0.2}^{+0.3}$ & $\cdots$ \\
\hline$k T_{\text {vnei }}(\mathrm{keV})$ & $\cdots$ & $\ldots$ & -0.2 & $0.70 \pm 0.02$ \\
\hline Norm $_{\text {vnei }}{ }^{\mathrm{d}}\left(10^{-3}\right)$ & $\ldots$ & $\ldots$ & $\ldots$ & $7.4_{-1.2}^{+1.0}$ \\
\hline$Z / Z_{\odot}$ & $\ldots$ & $\ldots$ & $\ldots$ & $0.004_{-0.003}^{+0.002}$ \\
\hline$O / O_{\odot}$ & $\ldots$ & $\ldots$ & $\ldots$ & $0.8_{-0.3}^{+0.4}$ \\
\hline$\tau\left(10^{11} \mathrm{~s} \mathrm{~cm}^{-3}\right)$ & $\ldots$ & $\ldots$ & $\ldots$ & $2.9_{-0.4}^{+0.8}$ \\
\hline$E_{\text {gauss }}(\mathrm{keV})$ & $0.643_{-0.003}^{+0.002}$ & $\ldots$ & $\ldots$ & $\ldots .4$ \\
\hline Norm $_{\text {gauss }}{ }^{\mathrm{e}}\left(10^{-3}\right)$ & $0.60_{-0.08}^{+0.09}$ & $\ldots$ & $\ldots$ & $\ldots$ \\
\hline XIS 1 normalization & 1.0 & 1.0 & 1.0 & 1.0 \\
\hline XIS 0 normalization & 0.92 & $0.92 \pm 0.03$ & $0.92 \pm 0.03$ & $0.92 \pm 0.03$ \\
\hline XIS 3 normalization & 0.87 & $0.85 \pm 0.03$ & $0.86 \pm 0.03$ & $0.86 \pm 0.02$ \\
\hline$\chi^{2}$ & 657.07 & 895.58 & 665.24 & 653.34 \\
\hline dof & 539 & 540 & 539 & 537 \\
\hline
\end{tabular}

Notes.

a All quoted uncertainties are $90 \%$ confidence intervals.

${ }^{\mathrm{b}} \mathrm{Norm}_{\mathrm{BB}}=\frac{L_{39}}{D_{10}^{2}}$, where $L_{39}$ is the bolometric luminosity of the source in units of $10^{39} \mathrm{erg} \mathrm{s}-1$, and $D_{10}$ is the distance to the source in units of $10 \mathrm{kpc}$.

${ }^{\mathrm{c}}$ Norm $_{\mathrm{br}}=\frac{3.02 \times 10^{-15}}{4 \pi D^{2}} \int n_{e} n_{i} d V$, where $D$ is the distance to the source in units of $\mathrm{cm}$ and $n_{e}$ and $n_{i}$ are the electron and ion number densities, respectively, in units of $\mathrm{cm}^{-3}$.

${ }_{\mathrm{d}}$ Norm $_{\mathrm{vapec}}=\frac{10^{-14}}{4 \pi D^{2}} \int n_{e} n_{i} d V$, where the parameters have the same meaning as in the bremsstrahlung model.

${ }^{\mathrm{e}}$ Norm $_{\text {gauss }}=$ Total photons $\mathrm{cm}^{-2} \mathrm{~s}^{-1}$ in the Gaussian line.

revealed that the source had increased in count rate by another order of magnitude, to around $0.4 \mathrm{cts} \mathrm{s}^{-1}$. The $0.3-10 \mathrm{keV}$ count rate remained high, although variable, until Day $\sim 180$, at which point it began to fade systematically over the following 200 days. By the time of the last monitoring observation with Swift on 2012 April 17, the source count rate was $\sim 0.07$ counts $\mathrm{s}^{-1}$.

\section{X-RAY SPECTRA OF THE 2011 OUTBURST OF T Pyx}

\subsection{A Deep View on Day 194 with Suzaku}

Although the Swift observations were carried out with high cadence, in general, the continuous observation durations were rather short and resulted in limited counting statistics for spectroscopy. The Suzaku observation on Day 194 resulted in $\sim 22,000$ counts between all three XIS units (Figure 2). We use this deep observation to characterize the X-ray emission in as much detail as possible, and to inform our model for the lower count statistic Swift data.

We modeled the spectra extracted from all three XIS units jointly in Xspec version 12.8.0m (Arnaud 1996), and present the resulting parameters in Table 1 . In all models, a constant was included in order to allow for uncertainty in the calibration of the relative effective areas of the three CCDs (typically of the order of 5\%-10\%).

To account for absorption by the interstellar medium (ISM) we use the tbnew model of Wilms et al. (2000), and assume the cross-sections of Verner et al. (1996) and the ISM abundances of Wilms et al. (2000). A range of values for the degree of interstellar absorption toward T Pyx have been given in the literature. Gilmozzi \& Selvelli (2007) find a value of
$E(B-V)=0.25 \pm 0.02$ from IUE ultraviolet spectra of T Pyx obtained during quiescence, which corresponds to a value of $N(H) \approx(1.3 \pm 0.1) \times 10^{21} \mathrm{~cm}^{-2}$ (assuming the correlation between $N(H)$ and $E(B-V)$ of Predehl \& Schmitt 1995). Shore et al. (2011) used diffuse interstellar bands observed during the 2011 outburst to determine $E(B-V)=0.49 \pm 0.17$ mag for T Pyx, implying $N(H) \approx(2.6 \pm 0.9) \times 10^{21} \mathrm{~cm}^{-2}$. Finally, Godon et al. (2014) determined an intermediate reddening value from UV spectroscopy, $E(B-V)=0.35$ mag.

The Suzaku spectrum is clearly complex. A distinct soft component is observed below $0.5 \mathrm{keV}$ and is most obvious in the XIS1 data, which has the highest sensitivity at low energies. A second, harder continuum component extends out to $\sim 6 \mathrm{keV}$. Finally, there is a rather striking resolved emission line at $E \approx 0.65 \mathrm{keV}$ that we identify as $\mathrm{O}$ VIII Ly $\alpha$. There are no obvious emission lines at any other location in the spectrum.

We begin our exploration of the spectrum with a simple model that accounts for the main features seen in the data: the sum of a blackbody, a thermal plasma, and a Gaussian emission line (model tbnew* (bb+brems+gauss) in Table 1). This model is not strictly physical, as the soft component is expected to show features typical of a white dwarf atmosphere and the plasma is expected to cool primarily through lines, but it does provide a useful starting point given the limited energy resolution of the spectrum. This simple model provides a reasonable fit to the data, with $\chi^{2} / v=1.26(v=539)$. The Gaussian line is clearly required to account for the feature at $\sim 0.65 \mathrm{keV}$. However, the best-fit energy of the emission line is found to be $0.643_{-0.003}^{+0.002} \mathrm{keV}$, implying that the O VIII line is redshifted by $\sim 5000 \mathrm{~km} \mathrm{~s}^{-1}$. This velocity is at odds with the results of Tofflemire et al. (2013), who reported that the O VIII Ly $\alpha$ line 
is blue-shifted by $\sim-400 \mathrm{~km} \mathrm{~s}^{-1}$ in a Chandra Low Energy Grating Spectrograph (LETG) observation taken a few months later (Day 210). The observed redshift is also larger than velocities derived from optical spectroscopy at any point during the outburst.

A careful examination of the residuals around the emission line in all three XIS units reveals a shift to lower energies in the XIS1 data, possibly indicating a problem with the energy calibration of the observation. At low energies $(<1 \mathrm{keV})$, uncertainties in the energy scale calibration come primarily from the determination of the CCD zero-level, and it is possible that this was more uncertain for the XIS1 CCD than the others. We investigated the presence of this energy offset in XIS1 relative to the other two CCDs using the gain fit command in XSpec. Fitting all three spectra independently with an absorbed blackbody model, we find evidence of an offset in the energy scale of XIS1 by approximately $-8 \mathrm{eV}$ relative to XIS0 and XIS3. Including this additional energy offset in the model fit decreases both the reduced $\chi^{2}$ value of the fit, and the residuals around the emission line in the XIS1 data. We therefore include an energy offset of $-8 \mathrm{eV}$ for the XIS1 spectrum in all of our fits moving forward. This energy offset is consistent with the estimated uncertainty in the energy scale below $1 \mathrm{keV}$ of $<10 \mathrm{eV}^{12}$ although it may indicate that the error in the energy scale determination for this observation was larger than typical values. Comparing fits with and without the offset, we find very little difference in the values associated with the blackbody and bremsstrahlung components, but the energy of the line shifts to higher energies with the inclusion of the energy offset. We therefore caution that the uncertainties in the line energy are larger than the statistical errors from the fit alone, likely of the order of $10 \mathrm{eV}$ (or $4500 \mathrm{~km} \mathrm{~s}^{-1}$ in velocity space).

The best-fit parameters obtained for the simple blackbody+thermal plasma+Gaussian line model, obtained with the inclusion of the additional XIS1 energy offset, are shown in Table 1. This model is presented in Figure 2, overplotted on the observed spectra. The best-fit $N(H)$ value for this model is only $(2.7 \pm 1.9) \times 10^{20} \mathrm{~cm}^{-2}$, much lower than the value expected from optical and UV estimates of reddening. The blackbody temperature, $k T_{\mathrm{BB}}$, is found to be $47 \pm 2 \mathrm{eV}(545,400 \pm$ $23,000 \mathrm{~K}, 90 \%$ confidence level), with the main constraint coming from the well-sampled Wien tail between 0.4 and $0.6 \mathrm{keV}$. The normalization of this component implies a blackbody luminosity of $\left(3.7_{-1.8}^{+3.5}\right) \times 10^{35} \mathrm{erg} \mathrm{s}^{-1}$. The plasma temperature is found to be $0.72 \pm 0.03 \mathrm{keV}$, or $(8.4 \pm 0.4) \times 10^{6} \mathrm{~K}$. The normalization of this component corresponds to a volume emission measure of $\mathrm{EM}_{V}=n^{2} V=\left(2.2_{-0.1}^{+0.2}\right) \times 10^{57} \mathrm{~cm}^{-3}$, or an X-ray luminosity of $\left(8.9_{-0.6}^{+1.0}\right) \times 10^{33} \mathrm{erg} \mathrm{s}^{-1}$.

We have accounted for the presence of the O VIII Ly $\alpha$ line in this simple model with an additional Gaussian. However it is more realistic to attempt to fit the data with a model that produces both continuum and line emission. Therefore, we also modeled the harder component with the APEC models of collisional ionization equilibrium plasmas (Foster et al. 2012). A solar abundance model gives a very poor fit to the data-the lines of He-like Si and S, as well as the Fe L-shell emission around $0.9 \mathrm{keV}$ are much too strong in the model compared to the data, while the O VIII Ly $\alpha$ line is too weak. If we allow the abundances of all metals to vary in tandem (while keeping their relative abundances at solar values), the model can achieve a good fit to the data with no residuals at the $\mathrm{Si}$ and $\mathrm{S}$ lines if

$\overline{12}$ http://web.mit.edu/iachec/meetings/2012/Presentations/Miller.pdf the best-fit metallicity is extremely subsolar $\left(Z \approx 0.01 Z_{\odot}\right)$. However, such a model still fails to reproduce the O vIII Ly $\alpha$ line observed in the data, and results in abundances seemingly at odds with the report of approximately solar abundances in the larger scale T Pyx ejecta (Williams 1982).

We obtain a better, but still inadequate, fit to the data if the abundance of $\mathrm{O}$ in the plasma is extremely supersolar, while assuming solar abundance for all other elements (model tbnew* (bb+vapec) $)_{a}$ in Table 1). Leaving both the $\mathrm{O}$ abundance of the plasma to vary freely and keeping all other abundances fixed at solar values relative to hydrogen, we find $\chi^{2} / v=1.7(v=540)$ for an oxygen abundance of $150_{-4}^{+6}$ times solar. The temperature of this plasma is similar to that found for the simple bremsstrahlung model $\left(k T_{\mathrm{br}}=0.70 \pm 0.02 \mathrm{keV}\right.$, or $\left.8.1 \times 10^{6} \mathrm{~K}\right)$. The temperature of the blackbody is slightly lower $\left(k T_{\mathrm{BB}}=35 \mathrm{eV}, T_{\mathrm{BB}}=406,000 \mathrm{~K}\right)$. The most marked difference is in the $N(H)$, which is found to be more in line with the optical and UV values, $N(H)=(2.0 \pm 0.02) \times 10^{21} \mathrm{~cm}^{-2}$.

The large oxygen abundance inferred by the strong emission line in our spectrum could indicate that the shocked material has been significantly polluted by material from the white dwarf. Interestingly, Tofflemire et al. (2013) find evidence for a strong overabundance (relative to solar) of $\mathrm{N}$ in their high spectral resolution Chandra/LETG data from Days 210 and 235; our earlier Suzaku data unfortunately do not have sufficient resolution at low energies to explore this finding. Both oxygen and nitrogen overabundance are suggestive of the presence of dredged-up white dwarf material in the thermal plasma.

We also tested an alternative possibility: that the X-rays come from a primarily oxygen-rich plasma that has very little metal content. We fit an absorbed blackbody + vapec model to the data, but this time allowed all elemental abundances to vary freely (model tbnew* (bb+vapec) ${ }_{b}$ in Table 1$)$. Given the lack of obvious spectral features, we kept the relative abundances of $\mathrm{He}, \mathrm{C}, \mathrm{N}$ and all metals with higher atomic numbers than $\mathrm{O}$ fixed at their solar ratios relative to $\mathrm{Fe}$, and allowed the abundance of Fe to vary freely. The abundance of oxygen was allowed to vary independently. This model results in a significantly better fit to the data than the previous model, with $\chi^{2} / v=1.2(v=539)$. The abundances of all metals other than $\mathrm{O}$ are essentially zero, and the abundance of oxygen relative to hydrogen is $\sim 60 \%$ enhanced relative to solar values. The temperatures of the two components are similar to those found for other models.

Alternatively, the bright oxygen line could be an indication that the plasma is underionized and has not yet come into collisional equilibrium (i.e., in non-equilibirum ionization; NEI). To test this scenario, we use the vnei model in XSpec. The overall spectrum is modeled as the sum of an absorbed blackbody plus NEI plasma (Model tbnew*(bb+vnei) in Table 1). Keeping all elemental abundances at their solar values, we find no fits with $\chi^{2} / v<1.7(v=538)$. Allowing the oxygen abundance to vary freely, but keeping all other elements fixed at solar does not improve the fit much $\left(\chi^{2} / v<1.6, v=537\right)$. Allowing all other elements to vary in tandem from their solar values, as we did for the vapec model above, does result in a better fit $\left(\chi^{2} / v=1.2\right.$, $v=537$ ), and results in a similar ratio of oxygen to other metals (see Table 1 for model parameters). The best-fit ionization age, $2.9 \times 10^{11} \mathrm{~s} \mathrm{~cm}^{-3}$, is close to the equilibrium value for a plasma with $k T=0.7 \mathrm{keV}$ (Smith \& Hughes 2010), suggesting that non-equilibrium ionization physics is not the primary reason for the strong oxygen relative to other metals.

In summary, the key results of our modeling of the Suzaku spectrum are (1) all models require both a soft and hard 
Table 2

Best-fit Model Parameters for Weekly Averaged Swift X-Ray Spectra ${ }^{a}$

\begin{tabular}{|c|c|c|c|c|c|c|c|c|c|c|}
\hline $\begin{array}{l}\text { Date Range } \\
\text { (UT) }\end{array}$ & $\begin{array}{c}\text { Time since } \\
\text { Outburst (Days) }\end{array}$ & $\begin{array}{c}N(H) \\
\left(10^{21} \mathrm{~cm}^{-2}\right)\end{array}$ & $\begin{array}{c}k T_{\mathrm{BB}} \\
(\mathrm{eV})\end{array}$ & $\operatorname{Norm}_{B B}{ }^{b}$ & $\begin{array}{c}k T_{\mathrm{br}} \\
(\mathrm{keV})\end{array}$ & $\begin{array}{c}\text { Norm }_{b r}{ }^{b} \\
\left(10^{-3}\right)\end{array}$ & $\begin{array}{l}E_{\text {gauss }} \\
(\mathrm{keV})\end{array}$ & $\begin{array}{c}\text { norm }_{\text {gauss }}{ }^{\mathrm{b}} \\
\left(10^{-3}\right)\end{array}$ & c-stat & D.O.F. \\
\hline 2011 Sep 3-9 & $142-149$ & $2.6_{-0.5}^{+0.5}$ & $32_{-3}^{+4}$ & $0.61_{-0.46}^{+1.76}$ & $1.20_{-0.13}^{+0.14}$ & $3.4_{-0.5}^{+0.6}$ & $0.666_{-0.014}^{+0.013}$ & $1.39_{-0.33}^{+0.59}$ & 268.1 & 267 \\
\hline 2011 Sep 10-16 & $149-156$ & $2.2_{-0.6}^{+0.6}$ & $40_{-5}^{+6}$ & $0.053_{-0.040}^{+0.257}$ & $1.21_{-0.17}^{+0.20}$ & $3.1_{-0.6}^{+0.7}$ & $0.666^{\mathrm{c}}$ & $0.94_{-0.60}^{+0.86}$ & 220.8 & 222 \\
\hline 2011 Sep 17-23 & $156-163$ & $1.5_{-0.4}^{+0.5}$ & $45_{-5}^{+6}$ & $0.013_{-0.008}^{+0.027}$ & $1.17_{-0.16}^{+0.15}$ & $3.3_{-0.5}^{+0.6}$ & $0.666^{\mathrm{c}}$ & $0.53_{-0.38}^{+0.50}$ & 273.7 & 243 \\
\hline 2011 Sep 24-30 & $163-170$ & $1.8_{-0.5}^{+0.5}$ & $43_{-5}^{+6}$ & $0.019_{-0.13}^{+0.013}$ & $1.09_{-0.15}^{+0.16}$ & $3.4_{-1.6}^{+0.8}$ & $0.666^{\mathrm{c}}$ & $0.79_{-0.46}^{+0.64}$ & 237.7 & 232 \\
\hline 2011 Oct $1-7$ & $170-177$ & $2.1_{-0.5}^{+0.7}$ & $39_{-5}^{+6}$ & $0.061_{-0.047}^{+0.312}$ & $1.01_{-0.07}^{+0.25}$ & $3.1_{-0.6}^{+0.9}$ & $0.666^{\mathrm{c}}$ & $1.50_{-0.71}^{+0.86}$ & 201.78 & 215 \\
\hline 2011 Oct 8-14 & $177-184$ & $1.4_{-0.4}^{+0.4}$ & $48_{-6}^{+6}$ & $0.0071_{-0.004}^{+0.018}$ & $1.00_{-0.14}^{+0.15}$ & $23.0_{-0.6}^{+0.7}$ & $0.666^{\mathrm{c}}$ & $0.46_{-0.35}^{+0.46}$ & 229.4 & 216 \\
\hline 2011 Oct $16-21$ & 184-191 & $0.7_{-0.3}^{+0.4}$ & $58_{-8}^{+7}$ & $0.0010_{-0.0005}^{+0.0019}$ & $0.92_{-0.14}^{+0.19}$ & $2.0_{-0.5}^{+0.6}$ & $0.666^{\mathrm{c}}$ & $<0.82$ & 161.0 & 189 \\
\hline 2011 Oct $23-29$ & $191-197$ & $1.2_{-0.6}^{+0.9}$ & $53_{-13}^{+13}$ & $0.0022_{-0.0017}^{+0.021}$ & $0.79_{-0.17}^{+0.27}$ & $2.3_{-0.9}^{+1.5}$ & $0.666^{\mathrm{c}}$ & $0.40_{-0.38}^{+0.64}$ & 129.8 & 146 \\
\hline 2011 Oct $30-$ Nov 5 & 197-204 & $1.2_{-0.7}^{+0.9}$ & $53_{-13}^{+16}$ & $0.0010_{-0.0008}^{+0.006}$ & $0.58_{-0.10}^{+0.16}$ & $2.6_{-1.1}^{+1.8}$ & $0.666^{\mathrm{c}}$ & $0.39_{-0.26}^{+0.47}$ & 122.7 & 148 \\
\hline
\end{tabular}

Notes.

a All quoted uncertainties are $90 \%$ confidence intervals.

b Norm $_{\mathrm{BB}}$, norm $\mathrm{br}$, and norm gauss as defined in Table 1.

c Parameter was fixed in model fit.

component to describe the emission, and (2) only a plasma that is significantly enhanced in oxygen relative to all other elements can reproduce the observed Suzaku spectrum at $E>0.5 \mathrm{keV}$.

\subsection{Spectral Fits from Swift}

To examine the time evolution of the distinct components we identified in the Suzaku spectrum, we extracted and modeled weekly averaged spectra from the Swift observations. The flux level and total number of counts before Day 142 are too low to allow useful spectra to be produced. Likewise, we find that after Day 206 the weekly spectra have $<100$ total counts, and so no stable model fits are found. We therefore restrict our spectroscopic analysis of the Swift data to weekly averaged spectra between Day 142 and Day 206 (2011 September 3-November 6). At each epoch we model the fit with the simple empirical tbnew* (bb+brems+gauss) model developed for the Suzaku data, i.e., the sum of a blackbody, a Gaussian emission line, and thermal bremsstrahlung absorbed by the interstellar medium. Visual inspection of the first few weekly spectra shows an enhancement of counts at the position of the $\mathrm{O}$ VIII line found in the Suzaku spectrum, justifying the inclusion of the Gaussian line component. We fixed the energy of the Gaussian line because of the lower number of counts in the Swift spectra, and allowed the line flux to vary freely. Given the uncertainties in the Suzaku energy calibration discussed in Section 4.1, we fixed the energy of the line to the best-fit line energy found from the Swift September 3-9 spectrum $(0.666 \mathrm{keV})$. Initial values for all other parameters are taken from the Suzaku best-fit model and then allowed to vary freely.

The resulting best-fit model parameter values and their $90 \%$ confidence interval uncertainties are shown in Figure 3 and listed in Table 2. Where the Swift and Suzaku observations overlap, Swift-derived parameters are consistent with measurements from the deeper Suzaku observation (Suzaku parameters from the tbnew* (bb+brems+gauss) model shown as red diamonds in Figures 3(d)-(h)). The only exception is the $N(H)$ value, which is considerably lower for the Suzaku best-fit model than the overlapping Swift data. However, we note that the error bars shown on this plot are the statistical uncertainties from the model fit only-they do not include a larger systematic uncertainty related to the choice of model (see Table 1 for range of $N(H)$ values found).
Over the $\sim 60$ days where T Pyx is X-ray bright, we see evolution in the spectrum and flux that is dominated by changes in the super-soft blackbody component (Figure 3(c)). The temperature of the thermal plasma component is roughly constant between Days 140 and 180, and then declines by a factor of $\sim 2$ by Day 206 (Figure 3(d)). The volume emission measure over the same time period does not vary significantly (Figure 3(e)), and so the decline in temperature results in a modest drop in flux from the hard X-ray component (see blue points in Figure 3(c)).

The drop in the total flux by a factor of $\sim 2$ between Day 142-206 is mainly attributable to the blackbody component (Figure 3(c)). The decline in the super-soft light curve can also be seen in Figure 3(h), where we see that the blackbody luminosity is lower after Day 175 than before. This decline in flux is despite a roughly constant value of the absorbing column density (Figure 3(f); consistent with the interstellar values estimated by Gilmozzi \& Selvelli 2007 and Shore et al. 2011) and a blackbody temperature evolution which shows no signature of decline (Figure $3(\mathrm{~g})$ ).

In all but one of the spectra, the flux of the emission line component is non-zero (Table 2). The flux appears to drop slightly in the first three weekly averaged spectra, and then rebrightens to its highest level in the October 1-7 spectrum. The line then fades again; we only obtain an upper limit to the line flux in the October 16-21 spectrum. The line flux is non-zero in the last two epochs, and $\sim 60 \%$ smaller compared to September measurements.

We stress that the X-ray properties shown in Figure 3(c)-(h) are only obtained during the plateau in the X-ray light curve, Days 142-206. The count rates measured before Day 117 and after Day $\sim 250$ are orders of magnitude lower, and so we cannot glean spectral information at this time due to the poor signal to noise in these observations. However, we can infer something about the spectral properties from the hardness ratio plot (Figure 3(b)). We choose $0.55 \mathrm{keV}$ as the break energy between the soft and hard bands, since our Suzaku spectral fits imply that all flux above this energy originates in the thermal plasma component. At very early times, the spectrum appears to be quite hard, particularly at the time of the first X-ray detections around Days 14-20. The spectrum then becomes softer as the source gets brighter. Although the error bars are quite large, the hardness ratio during the $\mathrm{X}$-ray rise is higher than at peak brightness, which suggests that the hard 
$\mathrm{X}$-ray component rose before the super-soft emission emerged. At late times, we observe a slight trend toward higher hardness ratios, presumably occurring as the super-soft continuum fades.

\section{THE ORIGIN OF HARD X-RAY EMISSION IN T Pyx}

The hard X-rays ( $E>1 \mathrm{keV})$, modeled in the previous section as thermal plasma and seen in all Swift/XRT detections, indicate the presence of a long-lived shock. Hard X-ray emission in novae can originate either in external material that is swept up and shocked by the nova ejecta (Sokoloski et al. 2006; Nelson et al. 2008, 2012), or in shocks within the ejecta themselves (O'Brien et al. 1994; Mukai \& Ishida 2001). In this section, we consider the possibility that the hard X-ray emission is produced by interaction between the nova ejecta and pre-existing circumbinary material. We will show that the timing of the hard $\mathrm{X}$-ray component rules out plausible models for circumbinary material around T Pyx as a significant source of X-rays. We therefore propose that an interaction within the 2011 ejecta, between two distinct episodes of ejection, explains the hard $\mathrm{X}$-rays.

The temperature associated with the thermal plasma emission encodes information about the kinematics of the shock. During the first week T Pyx is visible after solar conjunction (Days 142-148, the first Swift observations with good signal to noise; Table 2), the best-fit temperature of the hard X-ray emitting thermal plasma is $T_{\mathrm{br}} \approx 1.4 \times 10^{7} \mathrm{~K}$. This temperature is comparable to those measured in hard X-ray components of other novae (Lloyd et al. 1992; Balman et al. 1998; Mukai \& Ishida 2001), and can be translated into a rough estimate of the shock velocity using the Rankine-Hugoniot jump conditions for a strong shock:

$$
\begin{gathered}
v_{s}=\sqrt{\frac{16 k T_{\mathrm{br}}}{3 \mu m_{H}}} \\
\frac{v_{s}}{1000 \mathrm{~km} \mathrm{~s}^{-1}}=\sqrt{\frac{T_{\mathrm{br}}}{1.4 \times 10^{7} \mathrm{~K}}} .
\end{gathered}
$$

Here, $\mu$ is the mean molecular weight of the gas and $m_{H}$ is the mass of a hydrogen atom. We therefore estimate $v_{s} \approx 1000 \mathrm{~km} \mathrm{~s}^{-1}$ for the X-ray emitting shock in T Pyx.

The maximum velocity of the ejecta is a critical parameter for our models of shocked X-ray emission in T Pyx. We proceed with our discussion using $\mathrm{H} \beta$ measurements of the maximum blueshift from Paper I; these imply a maximum expansion velocity of $\sim 1900 \mathrm{~km} \mathrm{~s}^{-1}$ on Day 2 and $\sim 3000 \mathrm{~km} \mathrm{~s}^{-1}$ on Day 69 (see also Surina et al. 2014).

\subsection{Could Interaction with Circumbinary Material Produce the Hard X-Ray Component?}

A rich circumbinary medium is clearly present around $\mathrm{T}$ Pyx, in the form of a spatially resolved, clumpy $\mathrm{H} \alpha+[\mathrm{N}$ II] remnant (Williams 1982; Shara et al. 1997; Schaefer et al. 2010). Contini \& Prialnik (1997) propose that X-ray emission may be produced in T Pyx when ejecta from nova outbursts interact with this nebula. Balman (2010) and Balman et al. (2012) claimed a detection of spatial extension in X-ray images of T Pyx in quiescence, as might be expected for such interaction. Subsequently, however, this claim was called into question using re-analyzed high-resolution Chandra X-ray images (Montez et al. 2012).
Interaction between the 2011 ejecta and the spatially resolved nebula is simple to rule out with timescale arguments. The $\mathrm{H} \alpha+[\mathrm{N}$ II $]$ flux from the nebula peaks at 4 ."4, or $0.1 \mathrm{pc}$ (Shara et al. 1997). All nine of the nebula's "shells" proposed by Shara et al. (1997) have radii $>1^{\prime \prime}$, or $6 \times 10^{16} \mathrm{~cm}$. Similarly large radii, $2 \times 10^{17} \mathrm{~cm}$, are expected for the ejecta from the 1966 outburst, if they expand at $1900 \mathrm{~km} \mathrm{~s}^{-1}$. Assuming the 2011 ejecta are traveling at $1900-3000 \mathrm{~km} \mathrm{~s}^{-1}$, an encounter on Day 117 would imply that material from the 1966 ejection is traveling at just $20 \mathrm{~km} \mathrm{~s}^{-1}$. In the unlikely case that there are ejecta from 1966 traveling at such a low velocity, we would expect the shell of 1966 ejecta to be very thick (spanning velocities $20-3000 \mathrm{~km} \mathrm{~s}^{-1}$ ), and the shock interaction to be prolonged over years. Instead, the rise and plateau of the hard $\mathrm{X}$-ray light curve is contained to a relatively short period, Days 117-206 (Figure 3). Reasonable assumptions for the velocity and distribution of the 1966 ejecta therefore imply that they are much too distant to be encountered by the 2011 ejecta in the first year.

In addition to the ejecta from previous novae, there might be circumbinary material present at smaller radii, perhaps lost through a wind during the quiescent period, 1967-2011. Indeed, T Pyx seems to have an unusually high accretion rate for a CV, so it is not unreasonable to expect such mass loss (Selvelli et al. 2008; Schaefer et al. 2013; Godon et al. 2014). However, from UV spectroscopy during quiescence, there is no evidence of a strong or fast outflow from T Pyx (Gilmozzi \& Selvelli 2007).

Given the low inclination of T Pyx (Uthas et al. 2010), perhaps the easiest way to "hide" significant mass loss from the binary during quiescence is to have it leak out of an outer Lagrangian point and remain concentrated in the orbital plane; we might then expect mass loss to proceed at relatively modest speeds on order of the orbital velocity, $\sim 20 \mathrm{~km} \mathrm{~s}^{-1}$ (Uthas et al. 2010). The density of this equatorial circumbinary material should be highest near the binary, and fall off linearly with distance. This configuration of circumbinary material is in direct conflict with the primary late rise of the X-ray light curve. While the faint detections between Days 14-20 might be consistent with such an interaction, the X-ray non-detections between Days 20-117 imply a cavity surrounding $\mathrm{T}$ Pyx, extending between radii $\sim 4 \times 10^{14}-2 \times 10^{15} \mathrm{~cm}$. No simple picture of circumbinary material in T Pyx explains a cavity at these radii, surrounded by a dense shell. We therefore move on to consider an alternative explanation for the hard X-ray component in T Pyx that rises four months after outburst: shocks within the 2011 nova ejecta.

\subsection{Hard X-Rays Produced By Shocks Within the Ejecta}

Shocks may be produced within the nova ejecta themselves, if the speed of ejection increases with time after the thermonuclear runaway (O'Brien et al. 1994). In this case, the shock velocity $\left(v_{s}\right)$ is equal to $4 / 3$ the differential velocity between the inner and outer ejecta components. In fact, we see increasing outflow velocities in T Pyx over the first two months after outburst, as measured from optical spectroscopy (Paper I, Surina et al. 2014). The difference between the velocity of material ejection on Day 2 and Day 69, as measured from the $\mathrm{H} \beta$ profile ([3000-1900] km $\left.\mathrm{s}^{-1}=1100 \mathrm{~km} \mathrm{~s}^{-1}\right)$, is consistent with the interaction velocity estimated from the X-ray temperature $\left(3 / 4 v_{s} \approx 800 \mathrm{~km} \mathrm{~s}^{-1}\right)$.

Let us simplify the complex picture painted by optical spectroscopy into a cartoon scenario: one shell is expelled on Day 0 with a velocity of $\sim 1900 \mathrm{~km} \mathrm{~s}^{-1}$ and a second is expelled later on with a velocity of $3000 \mathrm{~km} \mathrm{~s}^{-1}$. The bright hard X-ray component appears in the Swift data on Day 117, and rises 
steeply until it reaches maximum on Day 142 and plateaus until Day $\sim 206$, after which the flux gradually fades. In our model, the period of the X-ray rise and plateau corresponds to the time it takes for the shock to plow through the first ejection (O'Brien et al. 1994); by Day 206, the first ejection would be completely shocked. Using these measured velocities and requiring that the outermost edges of the first and second ejections catch up with one another around Day 206, we find that the second ejection was released on Day 75 (in reasonable agreement with the increase of $\mathrm{H} \beta$ expansion velocity and the rise of radio flux density; Paper I, Surina et al. 2014).

In addition, the hard X-ray component shows a remarkably strong O VIII Ly $\alpha$ line in the Suzaku spectrum, implying that the shocked gas is overabundant in oxygen by a factor of $\sim 1-2$ orders of magnitude over solar values, as is commonly observed in nova ejecta (e.g., Gehrz et al. 1998). Such an unusually high oxygen abundance implies that the shocked material is nova ejecta (rather than solar abundance material which may have been stripped from the secondary star).

The neutral hydrogen column density shielding the shocked material decreases only a small amount, if at all, between Days 142 and 206, and it is not significantly higher than the ISM foreground value during this period (Figure 3(f)). Much more dramatic declines in $N(H)$ are seen in the hard X-ray components of novae like V1974 Cyg and V382 Vel (Balman et al. 1998; Mukai \& Ishida 2001). T Pyx's small decrease in $N(H)$ implies that the shock is not viewed through a dense absorbing screen and is not deeply embedded in the ejecta.

We conclude that the timing, temperature, and abundances of the bright hard X-ray component are all well described by a shock within the ejecta of the 2011 outburst.

\section{A SUPER-SOFT SOURCE EMERGES AROUND DAY 130 IN THE 2011 OUTBURST OF T Pyx}

We clearly observe the unveiling of the super-soft X-ray emission in the Swift observations of T Pyx. The super-soft phase is thought to begin when the nova ejecta have expanded enough to become optically thin to X-ray photons emanating from the still-burning shell on the surface of the white dwarf (e.g., Krautter 2008; Schwarz et al. 2011). We note that this super-soft turn-on is an observationally defined phenomenon; the hot white dwarf is almost certainly present at earlier times, but it is not observed because of the large absorbing column.

Our analysis of both the Swift and Suzaku spectra of T Pyx show clear evidence for a blackbody component with temperature of $35-45 \mathrm{eV}\left([4-5] \times 10^{5} \mathrm{~K}\right)$. The average bolometric luminosity of this blackbody between Days 143 and 150 is $\left(1.3_{-0.9}^{+8.0}\right) \times 10^{38} \mathrm{erg} \mathrm{s}^{-1}$, near the Eddington luminosity and typical of a still shell-burning white dwarf (we note that blackbody model fits to super-soft spectra are known to underestimate the temperature and overestimate the luminosity of the white dwarf photosphere, so the uncertainties are likely larger than the statistical errors from the model fit; Osborne et al. 2011b). The luminosity of the blackbody is variable and appears to decline over the period Days 143-206. Similar variations have been observed in other novae, such as V2491 Cyg, where the bolometric luminosity during the super-soft phase did not appear to be constant (Page et al. 2010).

The observed turn-on time of the super-soft source phase in novae has been identified as an important diagnostic of the ejecta mass, since more massive ejecta will take longer to thin out as they expand, eventually reaching a column density where soft X-rays produced at the white dwarf photosphere can be

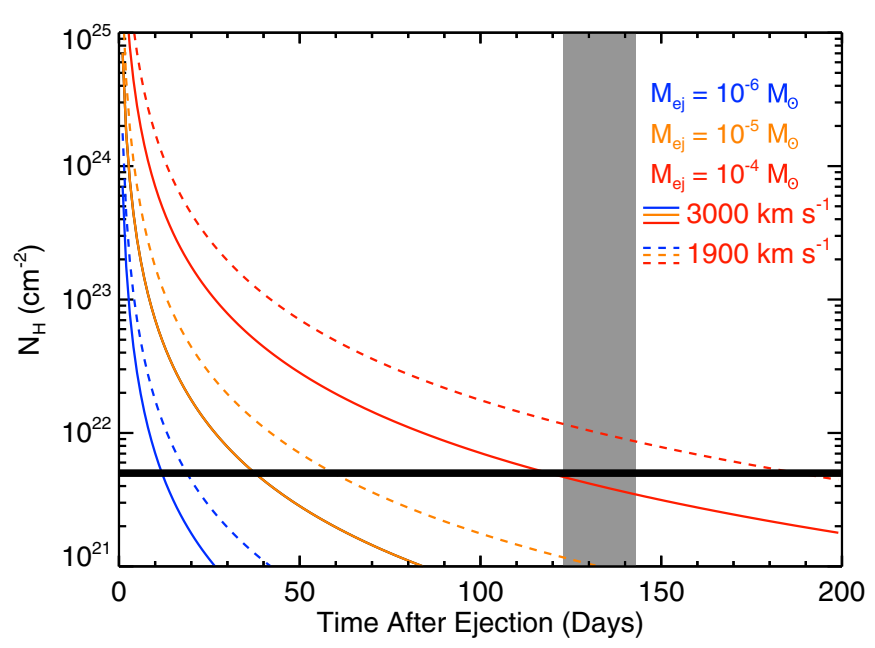

Figure 4. When the column density of nova ejecta drops sufficiently (to $\sim 5 \times 10^{21} \mathrm{~cm}^{-2}$; black solid line), the nuclear-burning white dwarf should become visible as a super-soft $\mathrm{X}$-ray source. The vertical gray region shows the plausible range of turn-on time for the super-soft phase in T Pyx, implying an ejecta mass $\gtrsim 10^{-5} M_{\odot}$. We overplot column density as a function of time for six different sets of ejecta parameters: those assuming $M_{\mathrm{ej}}=10^{-6} M_{\odot}$ (blue), $M_{\mathrm{ej}}=10^{-5} M_{\odot}$ (orange), and $M_{\mathrm{ej}}=10^{-4} M_{\odot}$ (red). Solid lines in blue, orange, and red represent ejecta with $v_{\max }=3000 \mathrm{~km} \mathrm{~s}^{-1}$, while dashed lines are for $v_{\max }=1900 \mathrm{~km} \mathrm{~s}^{-1}$.

(A color version of this figure is available in the online journal.)

transmitted through the ejecta. Schwarz et al. (2011) and Henze et al. (2011) explore the time elapsed between thermonuclear runaway and the observed turn-on of the super-soft source for large samples of novae. They find that super-soft turn-on time is correlated with the $M_{\mathrm{ej}} / v_{\max }$. Figure 4 shows the expected temporal evolution of the column density for shells ejected at Day 0 with a range of masses and two possible expansion velocities (chosen based on the $\mathrm{H} \beta$ line profiles of $\mathrm{T}$ Pyx at Days 2 and 69, respectively; Paper I). We assume that the shells have an $r^{-2}$ density profile and spherical symmetry that arise from a Hubble flow ejection with $v_{\min } / v_{\max }=0.2$ (see Paper I for details, also Seaquist \& Bode 2008). We estimate that $5 \times 10^{21} \mathrm{~cm}^{-2}$ is the maximum column density for which we could detect a super-soft component with temperature $k T<$ $50 \mathrm{eV}$ (horizontal black line).

The super-soft source is definitely observed on Day 143, the first observation after the solar gap. A careful examination of the data obtained on Days 120 and 123 constrain the earliest turn-on time to Day 123, when equal numbers of photons are detected above and below $0.5 \mathrm{keV}$, and a hint of an additional soft component can be seen in the spectrum. In Figure 4, we find that a turn-on time of 123 days corresponds to an ejected of mass of $\gtrsim$ few $\times 10^{-5} M_{\odot}$, assuming that the ejecta have been expanding since Day 0. If there is a two-month long delay in the ejection of the bulk of the mass, as suggested by the following section, the ejecta mass implied by the turn-on time can be slightly lower, but still $\gtrsim 10^{-5} M_{\odot}$. These mass estimates, while larger than traditionally assumed for T Pyx because of its short recurrence time (e.g., Contini \& Prialnik 1997; Schaefer et al. 2010), are compatible with estimates obtained from analysis of the 1966 outburst (Selvelli et al. 2008), radio light curves (Paper I), and the decrease of the binary period after the 2011 outburst (Patterson et al. 2013).

The blackbody temperature associated with the super-soft component is much lower than in other recurrent novae, implying a white dwarf in T Pyx which is significantly below the 
Chandrasekhar mass. More massive white dwarfs have smaller radii and surface areas, and so have higher temperature photospheres once they become super-soft sources (Sala \& Hernanz 2005; Wolf et al. 2013). The recurrent novae RS Oph and U Sco were both observed to have high blackbody temperatures during their super-soft phases (60-90 eV; Osborne et al. 2011b; Orio et al. 2013), as was the classical nova V2491 Cyg (Page et al. 2010). T Pyx is strikingly different, with a blackbody temperature of 30-50 eV, just half of what is measured in other recurrent novae (see also Tofflemire et al. 2013). Both the lower peak temperature and massive ejected shell are compatible with a nova outburst on a lower mass white dwarf $\left(\sim 1 M_{\odot}\right.$; Wolf et al. 2013).

The turn-off time of the super-soft source is also theoretically predicted to inversely scale with the white dwarf mass. The turnoff of the super-soft source refers to an abrupt decline in the blackbody luminosity, and likely marks the cessation of nuclear burning on the white dwarf surface, due to the exhaustion of the reservoir of hydrogen remaining after nova outburst. On a more massive white dwarf, the nuclear-burning luminosity is higher and the reservoir of hydrogen is less massive, implying a shorter duration for the super-soft phase (e.g., Sala \& Hernanz 2005).

We find evidence that the super-soft emission in T Pyx began to turn off around Day 180 (Section 4.2). This inference from the Swift data is in agreement with the report of a very lowluminosity blackbody component in the Chandra spectrum obtained on Day 210 (Tofflemire et al. 2013). Wolf et al. (2013) predict a stable shell-burning super-soft phase for $\gtrsim 1000$ days following a nova on a $1.0 M_{\odot}$ white dwarf; a super-soft duration of $\sim 200$ days is more consistent with a nova on a $1.15 M_{\odot}$ white dwarf. The rapid super-soft turn-off time in T Pyx therefore implies a somewhat more massive white dwarf, potentially at odds with the late observed turn-on time and the low effective temperature in the super-soft phase (e.g., Henze et al. 2014). However, as we show in the next section, mass loss from T Pyx is peculiar and complex; discrepancies in white dwarf mass constraints might be resolved if a larger fraction of the hydrogen envelope is expelled from T Pyx than theoretically predicted.

\section{X-RAY, OPTICAL, AND RADIO EVIDENCE FOR A STALLED EJECTION IN T Pyx}

In Section 5.2, we found that the hard X-rays in T Pyx are consistent with an interaction between two discrete shells: one which is triggered at Day 0 and expands at $1900 \mathrm{~km} \mathrm{~s}^{-1}$, and the other which begins expanding at $3000 \mathrm{~km} \mathrm{~s}^{-1}$ on Day $\sim 75$. This picture is also consistent with peculiarities seen at radio and optical wavelengths, as we discuss here.

The shapes of the radio and X-ray light curves are remarkably similar (Figure 1), which at first glance is surprising, as they are likely emitted by fundamentally different processes and regions. Both essentially show non-detections for the first couple months, then rise steeply (Figure 1). However, the quasi-coordinated rise is not a coincidence if the increases in both the radio and $\mathrm{X}$-ray regimes are tied to the expansion of the ejecta. The radio flux rises as the optically thick thermal ejecta expand and the emitting area increases in size. The expansion of the ejecta also leads to a drop in the column density shielding the hot white dwarf, revealing its super-soft X-ray emission. The hard X-ray emission is produced when fast-moving ejecta plow into slowermoving material. Based on arguments in Section 5, this material also appears to originate in the nova ejecta.

A detailed look at the two light curves reveals that the rises in the radio and X-ray are not perfectly synchronized. The first detection of the main radio rise was made on Day 67, while the first significant detection of the main X-ray rise did not take place until 1.5 months later, Day 117. The lag between the hard $\mathrm{X}$-ray and radio light curves cannot be explained if they share a common source, with the radio emission originating in the same shocked plasma as the $\mathrm{X}$-ray. In addition, the radio emission during Days 117-164 is far too bright and optically thick, and the temperature of the hard X-ray component is far too hot, to share a common source in the shocked gas (see the Appendix).

In Paper I, we showed that the peculiar radio light curve can be explained as either (1) a very cold ejection which is suddenly heated (requiring an increase in temperature by a factor of 25 in $\sim 19$ days) or (2) a delay in the expulsion of the bulk of the mass by $\sim 60$ days after the beginning of the optical rise. While both scenarios are consistent with the super-soft X-ray evolution of T Pyx, cooling and subsequent re-heating of the ejecta provides no natural explanation for the presence of a lateonset hard X-ray component that appears to be consistent with shock emission. Even when the white dwarf's photoionizing radiation can reach the outer ejecta and heat them, the radiation is too soft to result in X-rays with $E>1 \mathrm{keV}$. In contrast, the second scenario (a delay in ejecting the bulk of the mass from the binary; Figure 5) can self-consistently explain both the soft and hard X-ray components, along with the radio and optical evolution.

Our proposed multistage ejection scenario is illustrated in Figure 5. First, a shell constituting a small fraction $(\lesssim 10 \%)$ of the total envelope mass (shown in blue in Figure 5) is promptly expelled at $1900 \mathrm{~km} \mathrm{~s}^{-1}$ around the time of optical rise. Meanwhile, the remaining bulk of the accreted envelope (shown in red) puffs up into a quasi-hydrostatic envelope surrounding the binary for $\sim 60-70$ days. This stalled, massive shell, orbiting the central binary system as a common envelope-like structure with radius $\lesssim 10^{14} \mathrm{~cm}$, explains the bright, roughly constant plateau in the optical light curve and the early-time radio nondetections (Hachisu \& Kato 2004; Paper I). It is interesting to note that models of radio light curves imply that a low-mass ejection expelled on Day 0 might account for the sole radio detection at early times (measured on Day 17 and followed by a suite of non-detections on Days 31-48; Paper I), which is also coincident with the very early and short-lived detection of hard X-rays.

Optical spectra reveal that $\mathrm{T}$ Pyx originally resembled a $\mathrm{He} / \mathrm{N}$ nova but switched to a Fe II nova sometime between Day 2 and 10 (Ederoclite 2013; Williams 2013; see Williams 1992 and Williams 2012 for more interpretation of such spectral classifications). A wind with a low mass-loss rate but high velocity $\left(\dot{M}_{w} \approx 10^{-8} M_{\odot} \mathrm{yr}^{-1}, v_{w} \approx 2000 \mathrm{~km} \mathrm{~s}^{-1}\right)$ emanating from the puffed-up envelope may sustain the observed highvelocity wings on optical emission lines during Days $\sim 2-60$ (Surina et al. 2014) while remaining optically thin and below the detection limits in the radio on Days 31-48.

On Day 65, the optical spectrum of T Pyx was observed to transition back to a He/N classification (Ederoclite 2013), implying that the bulk of the ejected mass was now being lost in a discrete shell-like event (central panel of Figure 5). Around this time, the optical light curve begins to decline steeply and monotonically; we interpret this decline as the optical photosphere finally receding through the ejecta (Figure 1). In addition, the $\mathrm{H} \alpha$ line flux begins to systematically decrease (Surina et al. 2014), implying a drop in the density of the envelope. It is also around Day 65 that we see the radio light curve begin to rise, implying that the envelope is now growing in 


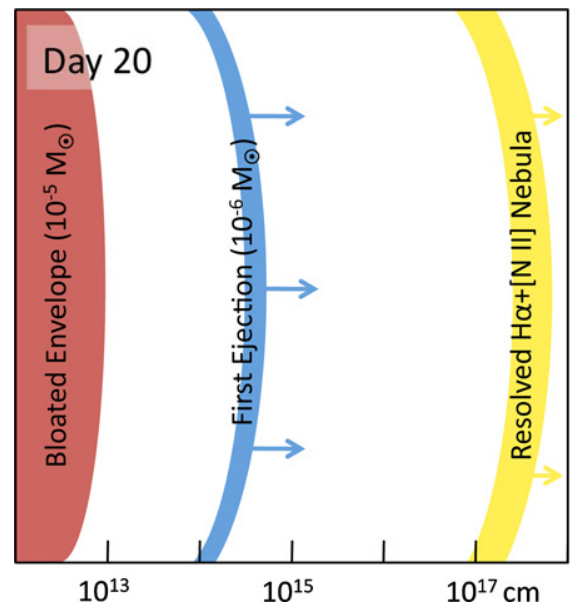

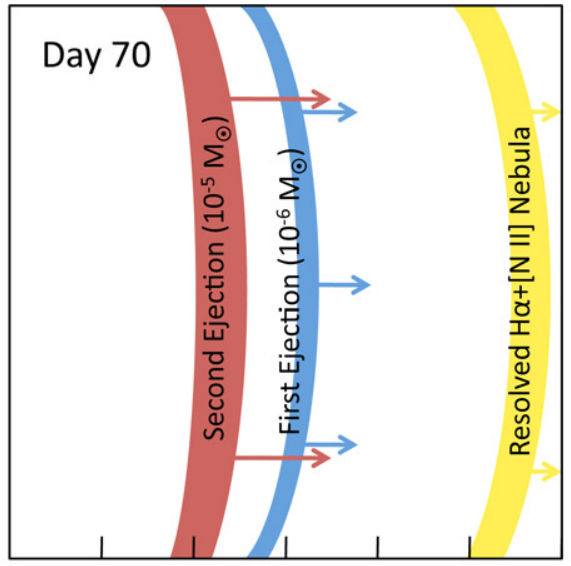

$10^{13}$

$10^{15}$

$10^{17} \mathrm{~cm}$

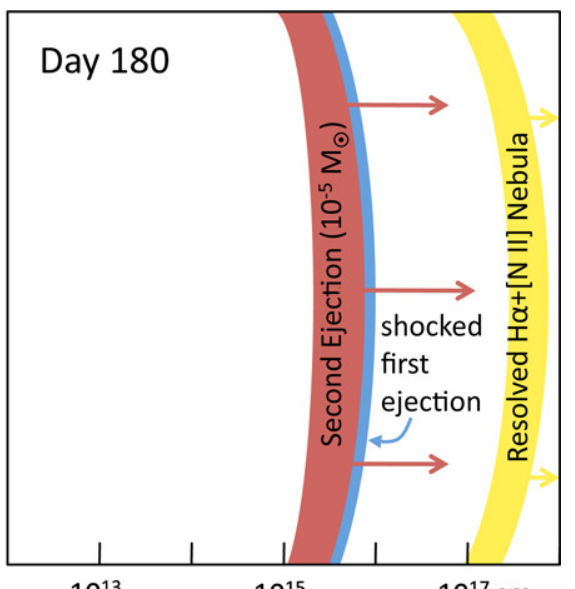

$10^{13}$

$10^{15}$

$10^{17} \mathrm{~cm}$

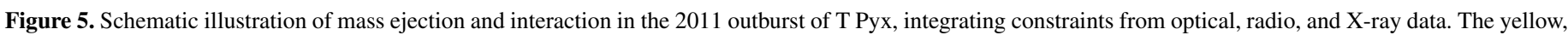

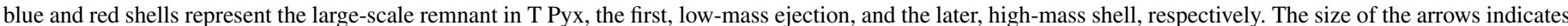

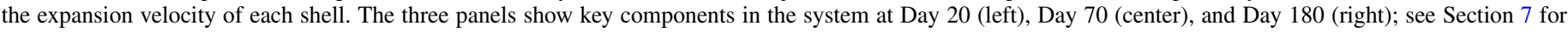
more details.

(A color version of this figure is available in the online journal.)

size (Paper I). Lastly, in our internal shock model for the hard $\mathrm{X}$-ray emission from T Pyx, we calculated that the faster moving ejection was likely expelled around Day 75 (Section 5.2; not precisely matching transitions at other wavelengths, but reasonably close given the uncertainties involved in our interpretation of the hard X-ray light curve). The agreement in timing from optical, radio, and X-ray tracers is remarkable; all imply that the bulk of the ejecta mass was not expelled from the environs of $\mathrm{T}$ Pyx until $\sim 2$ months after the thermonuclear runaway.

The physical cause of this stalled expansion remains unclear. Other novae have shown similar plateaus around optical maximum, with the duration of the plateau widely varying between sources, from a few to hundreds of days (e.g., Kato \& Hachisu 2011). However, most are not graced with rich multi-wavelength data sets like the community obtained for T Pyx in 2011, so these plateaus have proved difficult to interpret. Friedjung (1992) noted that during the plateau in HR Del, the optical spectrum showed little evidence of sustained strong mass loss as seen in other novae; it was in fact more consistent with an almost stationary atmosphere. The author proposed that the nova outburst in this system was initially not strong enough to eject the accreted envelope, and instead settled into a quasi-static configuration with little mass loss. No explanation was offered, however, for the transition to the subsequent phase of significant mass loss. Hachisu \& Kato (2004) concurred that plateaus around optical maximum could be interpreted as a static phase of the outburst, with an inflated but stable white dwarf photosphere formed after the onset of the thermonuclear runaway.

In later work, Kato \& Hachisu (2011) proposed that the interaction of the companion star with the hydrostatic envelope could provide an additional source of energy, empowering the outburst to transition from a stable to a mass-losing configuration. Kato \& Hachisu (2011) find that this mechanism should be most efficient in very short period binaries, like T Pyx, where the companion becomes deeply embedded in the nova envelope. In addition, Livio et al. (1990) and Lloyd et al. (1997) find that novae which undergo such a common-envelope-like phase may display ring-like morphologies in their ejecta, aligned with the orbital plane; Sokoloski et al. (2013) find hints of a ring structure in the $\mathrm{H} \alpha+[\mathrm{N}$ II $]$ nebula surrounding T Pyx.
A different explanation for a delayed ejection from $\mathrm{T}$ Pyx is proposed by Williams (2012); he suggests that a significant fraction of the mass loss may have its source in the binary companion star, rather than the white dwarf. If the nova explosion sweeps up or ablates material from the companion star, some of the peculiarities of T Pyx, like its hyper-hybrid spectral classification, could be explained. Detailed modeling of the interaction of the nova event with the companion star is required to assess the energetics, mass budget, and timescale of the interaction and evaluate if mass loss from the companion star might help explain the 2011 outburst of T Pyx.

Even more speculatively, peculiarities like multiple and delayed ejections might be explained if the nuclear-burning rate on the white dwarf surface fluctuates with time. Novae show an astounding diversity of light curves (Strope et al. 2010), some of which are difficult to explain with a single thermonuclear runaway. Indeed, nuclear-driven X-ray bursts on neutron stars sometimes display multiple thermonuclear runaways in the course of a single outburst (e.g., in't Zand et al. 2003). While multiple thermonuclear runaways are not theoretically predicted in a single nova outburst, they cannot be ruled out by current observations.

Considering all the available data in tandem, we favor a delayed ejection as the primary explanation for the multiwavelength evolution of T Pyx, because this scenario can selfconsistently explain the late and steep rise in the radio and X-ray light curves, the slow evolution of the optical light curve, and the unusually complex optical spectral evolution of T Pyx. The physical mechanism driving this late-time mass ejection remains unclear.

\section{CONCLUSIONS}

In our analysis of Swift and Suzaku data covering the 2011 outburst of the recurrent nova T Pyx, we detect two distinct components of X-ray emission: a super-soft component associated with the photosphere of the nuclear-burning white dwarf, and a hard component associated with shocked thermal gas.

The super-soft X-ray component becomes detectable between Days 123-143, implying an ejection which is surprisingly massive for a recurrent nova $\left(\gtrsim 10^{-5} M_{\odot}\right)$, but consistent with other recent constraints for T Pyx (Selvelli et al. 2008; 
Patterson et al. 2013, Paper I). In addition, the temperature of the super-soft source is relatively cool (30-50 eV), implying that the white dwarf in T Pyx is significantly below the Chandrasekhar mass. T Pyx therefore inhabits a poorly explored corner of nova parameter space, where its unusually high accretion rate, rather than an unusually massive white dwarf, drives it to have a short nova recurrence time.

A hard X-ray component is also detected in all epochs with sufficient signal to noise for spectral analysis (Days 142-206). Hard X-rays are relatively common in novae, and are postulated to originate in shocks internal to the nova ejecta (O'Brien et al. 1994; Krautter 2008). The hard X-rays in T Pyx, however, are accompanied by an unusually strong O VIII Ly $\alpha$ emission line, which can only be fit if the thermal plasma has a highly supersolar abundance of oxygen. Such high oxygen abundances in the nova ejecta require the dredge-up of significant amounts of white dwarf materials during the nova event (contrary to some models of recurrent novae; e.g., Starrfield et al. 2012). Similar conclusions can be reached from analysis of Chandra LETG spectra, which find a factor of $\sim 15$ overabundance of nitrogen in the ejecta, relative to solar (consistent with enrichment by CNO-processed white dwarf material; Tofflemire et al. 2013).

In addition, we note the appearance of a faint, hard $\mathrm{X}$-ray component at early times with an unknown origin. This component was only detectable by Swift/XRT in time-averaged spectra spanning Days 14-20, and its low signal to noise precludes detailed spectral analysis. However, we note a single coincident radio detection with the VLA at $33 \mathrm{GHz}$ on Day 17 (Paper I). The nature of this component remains a mystery, although it may be linked to the first low-mass ejection from T Pyx on Day 0 (Figure 5).

As measured from optical emission line profiles, the expansion velocity of ejecta in T Pyx increases by $50 \%$ during the first two months of outburst (Paper I, Surina et al. 2014); we find that this variation, and subsequent interaction within the ejecta, can naturally explain the temperature and light curve of the hard X-ray component. In Section 7, we present a cartoon picture that can self-consistently explain the soft and hard X-ray evolution, the radio light curve, the optical spectral evolution, and the optical light curve. A shell of material is expelled on Day 0 at $1900 \mathrm{~km} \mathrm{~s}^{-1}$, and a second episode of mass ejection is released on Day $\sim 65$ at $3000 \mathrm{~km} \mathrm{~s}^{-1}$ (Figure 5). While the first ejection produces clearer signatures at optical wavelengths, the second ejection accounts for the bulk of the expelled mass (as implied by the small absorbing column in the hard X-ray component and the bright but delayed maximum in the radio light curve). We do not yet understand the physical mechanism that leads to the bulk of the nova mass stalling out and lingering around the binary for two months before finally being expelled, but T Pyx now joins a significant and growing sample of novae which show evidence for complex, multi-phase mass ejection (see Lynch et al. 2008; Krauss et al. 2011; Williams 2012 for other examples).

We are grateful to R. Williams, A. Ederoclite, M. Bode, R. Smith, and U. Munari for useful conversations. We thank the Suzaku mission for the generous allocation of target-ofopportunity time to observe T Pyx. We also thank Neil Gehrels and the Swift mission team for their support of the target-ofopportunity program for this nova. We acknowledge with thanks the variable star observations from the AAVSO International Database contributed by observers worldwide and used in this research. This work made use of the HEASARC archive, data supplied by the UK Swift Science Data Centre at the University of Leicester, and observations obtained with the Suzaku satellite, a collaborative mission between the space agencies of Japan (JAXA) and the USA (NASA). The National Radio Astronomy Observatory is a facility of the National Science Foundation operated under cooperative agreement by Associated Universities, Inc. L. Chomiuk is a Jansky Fellow of the National Radio Astronomy Observatory. J. Osborne and K. Page acknowledge the support of the UK Space Agency. J. L. Sokoloski and J. Weston acknowledge support from NSF award AST-1211778.

\section{Facilities: Swift, Suzaku, VLA, AAVSO \\ APPENDIX \\ PREDICTING RADIO LUMINOSITY OF THE HARD X-RAY EMITTING GAS}

Here we investigate if the same thermal gas which emits hard X-rays in T Pyx can account for a significant portion of the observed radio flux. Take for example Day 156 (2011 September 17), a date during the optically thick rise of the radio light curve when both VLA radio and Swift X-ray observations were obtained. The X-ray observations imply a volume emission measure of $2.36 \times 10^{57} \mathrm{~cm}^{-3}$ and a temperature of $1.2 \mathrm{keV}$ $\left(1.4 \times 10^{7} \mathrm{~K}\right)$.

Assuming a spherical shell with radius of $1900 \mathrm{~km} \mathrm{~s}^{-1} \times 156$ Days $=2.6 \times 10^{15} \mathrm{~cm}$ and thickness of $10 \%$ the radius, this $\mathrm{EM}_{V}$ corresponds to a number density of $n=3 \times 10^{5} \mathrm{~cm}^{-3}$. The path-length emission measure, $\mathrm{EM}=\int n^{2} d l$, determines the radio optical depth and therefore radio luminosity; here it is $\mathrm{EM}=2 \times 10^{7} \mathrm{~cm}^{-6} \mathrm{pc}$. The optical depth of radio emission is:

$$
\tau_{\nu}=0.08235\left(\frac{\mathrm{EM}}{\mathrm{cm}^{-6} \mathrm{pc}}\right)\left(\frac{T_{\mathrm{sh}}}{\mathrm{K}}\right)^{-1.35}\left(\frac{v}{\mathrm{GHz}}\right)^{-2.1}
$$

(Seaquist \& Bode 2008). Therefore, the radio optical depth of the shocked gas is $\tau_{v}=2 \times 10^{-6}$ at $12.6 \mathrm{GHz}$, much less than unity and implying that the radio emission is completely optically thin. The radio flux density expected from this shocked gas is:

$$
\left(\frac{S_{v}}{\mathrm{mJy}}\right)=1.39 \times 10^{8}\left(\frac{T_{\mathrm{sh}}}{\mathrm{K}}\right)\left(\frac{v}{\mathrm{GHz}}\right)^{2}\left(\frac{R_{\mathrm{sh}}}{D}\right)^{2}\left(1-e^{-\tau_{v}}\right),
$$

where $R_{\mathrm{sh}}$ is the radius of the shock and $D$ is the distance to the emitting body, both in $\mathrm{cm}$. From the hard X-ray emission measured on Day 156, we therefore predict a $12.6 \mathrm{GHz}$ flux density of $0.02 \mathrm{mJy}$. On that day, we instead measured a $12.6 \mathrm{GHz}$ flux density from T Pyx of $11 \mathrm{mJy}$, a factor of 550 brighter than expected from the X-ray emitting shocked gas.

Similar results hold between Days 67-164; the shocked material that produces the hard X-rays (or upper limits on the hard X-rays) is not sufficient to account for the radio emission. The bulk of the radio emission must be coming from another source. This estimate underlines the well-known result that warm $10^{4} \mathrm{~K}$ gas is an efficient emitter at radio wavelengths, while the hot $>10^{6} \mathrm{~K}$ gas which efficiently emits at X-ray wavelengths is usually optically thin and relatively faint in the radio.

\section{REFERENCES}

Abdo, A. A., Ackermann, M., Ajello, M., et al. 2010, Sci, 329, 817

Arnaud, K. A. 1996, in ASP Conf. Ser. 101, Astronomical Data Analysis Software and Systems V, ed. G. H. Jacoby \& J. Barnes (San Francisco, CA: ASP), 17 
Balman, S. 2010, MNRAS, 404, L26

Balman, Ş., Krautter, J., \& Oegelman, H. 1998, ApJ, 499, 395

Balman, S., Starrfield, S., Drake, J. J., et al. 2012, ATel, 3915, 1

Cash, W. 1979, ApJ, 228, 939

Chesneau, O., Meilland, A., Banerjee, D. P. K., et al. 2011, A\&A, 534, L11

Contini, M., \& Prialnik, D. 1997, ApJ, 475, 803

De Gennaro Aquino, I., Shore, S. N., Schwarz, G. J., et al. 2014, A\&A, 562, A28

Ederoclite, A. 2013, arXiv:1304.1305

Evans, A., Gehrz, R. D., Helton, L. A., et al. 2012, MNRAS, 424, L69

Evans, P. A., Beardmore, A. P., Page, K. L., et al. 2009, MNRAS, 397, 1177

Foster, A. R., Ji, L., Smith, R. K., \& Brickhouse, N. S. 2012, ApJ, 756, 128

Friedjung, M. 1992, A\&A, 262, 487

Gehrz, R. D., Truran, J. W., Williams, R. E., \& Starrfield, S. 1998, PASP, 110, 3

Gilmozzi, R., \& Selvelli, P. 2007, A\&A, 461, 593

Godon, P., Sion, E. M., Starrfield, S., et al. 2014, ApJL, 784, L33

Hachisu, I., \& Kato, M. 2004, ApJL, 612, L57

Henze, M., Pietsch, W., Haberl, F., et al. 2011, A\&A, 533, A52

Henze, M., Pietsch, W., Haberl, F., et al. 2014, A\&A, 563, A2

Hill, A. B., Cheung, C. C., \& Jean, P. 2013, in Proc of Recontres de Moriond: Very High Energy Phenomena in the Universe, ed. E. Augé, J. Dumarchez, \& J. Trân Thanh Vân (Paris: ARISF)

Imamura, K., \& Tanabe, K. 2012, PASJ, 64, L9

in't Zand, J. J. M., Strohmayer, T. E., Markwardt, C. B., \& Swank, J. 2003, A\&A, 409,659

Kato, M., \& Hachisu, I. 2011, ApJ, 743, 157

Knigge, C. 2011, in ASP Conf. Ser. 447, Evolution of Compact Binaries, ed. L. Schmidtobreick, M. R. Schreiber, \& C. Tappert (San Francisco, CA: ASP), 3

Knigge, C., King, A. R., \& Patterson, J. 2000, A\&A, 364, L75

Krauss, M. I., Chomiuk, L., Rupen, M., et al. 2011, ApJL, 739, L6

Krautter, J. 2008, in Classical Novae, ed. M. F. Bode \& A. Evans (2nd ed.; Cambridge Astrophysics Series, No. 43, Cambridge: Cambridge Univ. Press), 232

Kuulkers, E., Orio, M., Osborne, J. P., et al. 2011a, ATel, 3647, 1

Kuulkers, E., Page, K. L., Ness, J.-U., et al. 2011b, ATel, 3285, 1

Livio, M., Shankar, A., Burkert, A., \& Truran, J. W. 1990, ApJ, 356, 250

Lloyd, H. M., O’Brien, T. J., \& Bode, M. F. 1997, MNRAS, 284, 137

Lloyd, H. M., O'Brien, T. J., Bode, M. F., et al. 1992, Natur, 356, 222

Lynch, D. K., Woodward, C. E., Gehrz, R., et al. 2008, AJ, 136, 1815

Montez, R., Sokoloski, J. L., \& Nelson, T. 2012, ATel, 4097, 1

Mukai, K., \& Ishida, M. 2001, ApJ, 551, 1024

Mukai, K., Orio, M., \& Della Valle, M. 2008, ApJ, 677, 1248

Nelson, T., Chomiuk, L., Roy, N., et al. 2014, ApJ, 785, 78

Nelson, T., Donato, D., Mukai, K., Sokoloski, J., \& Chomiuk, L. 2012, ApJ, 748,43

Nelson, T., Orio, M., Cassinelli, J. P., et al. 2008, ApJ, 673, 1067
Ness, J.-U., Osborne, J. P., Dobrotka, A., et al. 2011, ApJ, 733, 70

Ness, J.-U., Osborne, J. P., Henze, M., et al. 2013, A\&A, 559, A50

O'Brien, T. J., Lloyd, H. M., \& Bode, M. F. 1994, MNRAS, 271, 155

Orio, M. 2012, BASI, 40, 333

Orio, M., Behar, E., Gallagher, J., et al. 2013, MNRAS, 429, 1342

Osborne, J. P., Beardmore, A. P., Page, K. L., et al. 2011a, ATel, 3549, 1

Osborne, J. P., Page, K. L., Beardmore, A. P., et al. 2011b, ApJ, 727, 124

Page, K. L., Osborne, J. P., Evans, P. A., et al. 2010, MNRAS, 401, 121

Patterson, J., Oksanen, A., Monard, B., et al. 2013, arXiv:1303.0736

Predehl, P., \& Schmitt, J. H. M. M. 1995, A\&A, 293, 889

Rauch, T., Orio, M., Gonzales-Riestra, R., et al. 2010, ApJ, 717, 363

Sala, G., \& Hernanz, M. 2005, A\&A, 439, 1061

Schaefer, B. E. 2010, ApJS, 187, 275

Schaefer, B. E., Landolt, A. U., Linnolt, M., et al. 2013, ApJ, 773, 55

Schaefer, B. E., Pagnotta, A., \& Shara, M. M. 2010, ApJ, 708, 381

Schwarz, G. J., Ness, J.-U., Osborne, J. P., et al. 2011, ApJS, 197, 31

Seaquist, E. R., \& Bode, M. F. 2008, in Classical Novae, ed. M. F. Bode \& A. Evans (2nd ed.; Cambridge Astrophysics Series, No. 43, Cambridge: Cambridge Univ. Press), 141

Selvelli, P., Cassatella, A., Gilmozzi, R., \& González-Riestra, R. 2008, A\&A, 492, 787

Shara, M. M., Zurek, D. R., Williams, R. E., et al. 1997, AJ, 114, 258

Shore, S. N., Augusteijn, T., Ederoclite, A., \& Uthas, H. 2011, A\&A, 533, L8

Shore, S. N., Schwarz, G. J., De Gennaro Aquino, I., et al. 2013, A\&A, 549, A140

Smith, R. K., \& Hughes, J. P. 2010, ApJ, 718, 583

Sokoloski, J. L., Crotts, A. P. S., Lawrence, S., \& Uthas, H. 2013, ApJL, 770, L33

Sokoloski, J. L., Luna, G. J. M., Mukai, K., \& Kenyon, S. J. 2006, Natur, 442,276

Starrfield, S., Timmes, F. X., Iliadis, C., et al. 2012, BaltA, 21, 76

Strope, R. J., Schaefer, B. E., \& Henden, A. A. 2010, AJ, 140, 34

Surina, F., Hounsell, R. A., Bode, M. F., et al. 2014, AJ, 147, 107

Tatischeff, V., \& Hernanz, M. 2007, ApJL, 663, L101

Tofflemire, B. M., Orio, M., Page, K. L., et al. 2013, ApJ, 779, 22

Uthas, H., Knigge, C., \& Steeghs, D. 2010, MNRAS, 409, 237

Verner, D. A., Ferland, G. J., Korista, K. T., \& Yakovlev, D. G. 1996, ApJ, 465,487

Waagan, E., Linnolt, M., Bolzoni, S., et al. 2011, CBET, 2700, 1

Williams, R. 2012, AJ, 144, 98

Williams, R. 2013, AJ, 146, 55

Williams, R. E. 1982, ApJ, 261, 170

Williams, R. E. 1992, AJ, 104, 725

Wilms, J., Allen, A., \& McCray, R. 2000, ApJ, 542, 914

Wolf, W. M., Bildsten, L., Brooks, J., \& Paxton, B. 2013, ApJ, 777, 136

Yaron, O., Prialnik, D., Shara, M. M., \& Kovetz, A. 2005, ApJ, 623, 398 